\title{
Gender and autistic personality traits predict perspective-taking ability in typical adults
}

Tad T. Brunyé, Tali Ditman, Grace E. Giles, Caroline R. Mahoney, Klaus Kessler and Holly A. Taylor

\section{Introduction}

The ability to imagine oneself in another person's shoes, or visual perspective taking (VPT), allows us to temporarily experience the world from an imagined perspective. This ability is critical to everyday social interaction, and considered an important precursor to empathizing with and understanding another's psychological state (Hamilton, Brindley, \& Frith, 2009). Despite its importance and ubiquity in everyday experience, surprisingly few studies have examined individual differences that predict the ability to adopt another's perspective (e.g., Eisenberg and Lennon, 1983, Leith and Baumeister, 1998 and Tangney, 1995). The present study further elucidates the individual differences that may be associated with perspective-taking abilities by examining whether sub-clinical autistic personality traits in typical adults predict perspective-taking ability.

Visual perspective-taking involves two separable cognitive, neural, and developmental mechanisms: VPT-1 and VPT-2 (Flavell, Green, \& Flavell, 1986). The first level of VPT is involved in determining whether a person can see an object by following their line of sight (VPT-1); for instance, understanding whether an individual can or cannot see an occluded object. The second level of VPT involves the ability to adopt another's perspective in order to understand their unique vantage point (VPT-2); for instance, understanding that an object looks different from another person's perspective, or that it is to their right or left (Davis, 1983). This ability is generally more cognitively demanding than VPT-1, tends to develop later in childhood, and involves somewhat different cortical networks relative to VPT-1 (e.g., Ohnishi et al., 2004 and Ruby and Decety, 2001). Some recent work suggests that the ability to adopt another's perspective is separable from more general spatial abilities, such as mental rotation or the ability to manipulate objects (Hegarty and Waller, 2004 and Kozhevnikov and Hegarty, 2001). When considered in isolation from these more general visuospatial abilities, only a few studies have identified individual differences that specifically predict perspective-taking ability. For instance, Leith and Baumeister (1998) found that guilt-prone individuals showed greater perspective-taking abilities during a task that involved describing interpersonal conflict experiences from another's perspective. Some other work suggests that females generally show greater perspective-taking abilities than males (Eisenberg \& Lennon, 1983); even by 3 years of age girls show a greater ability than boys to adopt another's perspective to infer what they are thinking or intending (Happe, 1995). Even in adults, perspective-taking surveys consistently demonstrate higher self-rated perspective-taking and empathizing in female versus male respondents (Davis, 1980 and Davis and Kraus, 1991). This gender difference may reflect females being thought of as having generally more empathizing and males more systemizing personalities, the latter of which is frequently associated with the autism spectrum (Baron-Cohen et al., 2001b and Kessler, in press). Supporting this contention, the prevalence of autism spectrum disorder diagnosis is four times higher in boys than girls (e.g., Kogan et al., 2009).

Some recent research suggests that VPT-2 is specifically impaired in children with autism spectrum disorders (ASDs) relative to a closely matched group of typically developing children (Hamilton et al., 
2009). The ability to perform tasks demanding VPT-2 is often linked to mentalizing, which involves imagining oneself in another's shoes to understand their beliefs and predict behavior. Studies examining mentalizing in autism have consistently shown impairment in the ability to understand others'beliefs, and neuroimaging studies have demonstrated that mentalizing and VPT-2 tasks may share overlapping neural resources (e.g., Aichhorn, Perner, Kronbichler, Staffen, \& Ladurner, 2006). In contrast, studies examining VPT-1 abilities in ASDs demonstrate that these tasks are performed at a level similar to that seen in typical development (e.g., Baron-Cohen, 1989).

Recent theoretical enumerations suggest that the characteristic impairments seen in ASDs are thought to lie on a continuum of social and communication abnormalities that extends into the typical population (Baron-Cohen et al., 2001b). This conceptualization proposes a more quantitative than categorical or qualitative approach to both diagnosis and theoretical understanding of ASDs (Frith, 2001). The autism-spectrum quotient (AQ; Baron-Cohen et al., 2001b) allows for a nondiagnostic assessment of sub-clinical autistic traits, with higher scores indicating a greater degree of ASD-characteristic traits. The AQ has been validated in international samples of typical adults and individuals with Asperger syndrome and high functioning autism, demonstrating both high reliability and criterion validity (e.g., Wakabayashi, Baron-Cohen, Wheelwright, \& Tojo, 2006).

Though there is a lack of research specifically testing whether individual differences in autistic traits measured with the $A Q$ may predict visual perspective-taking abilities, there is some strong evidence to believe that this relationship may exist, specifically with regard to VPT-2 performance. First, as detailed above, some recent work suggests that children diagnosed with ASD show impairment during tasks challenging VPT-2, relative to matched controls (Hamilton et al., 2009). Second, individual differences in autistic personality traits among typical adults predict performance on some tasks impaired in autism, such as empathizing with others as a function of facial expression (e.g., Baron-Cohen, Wheelwright, Hill, Raste, \& Plumb, 2001a). Perspective-taking is also thought to be the primary cognitive component responsible for successful empathizing (e.g., Underwood \& Moore, 1982); it could be the case that variation in visual and/or cognitive perspective-taking as a function of autism spectrum traits might prove at least partially responsible for failed empathizing. Third, high autistic personality traits (using the $A Q$ ) have also been shown to predict brain structure and function, with higher $A Q$ scores predicting decreased white matter volume and increased cortical deactivation in brain areas considered critical to understanding social cues, such as the posterior superior temporal sulcus (von dem Hagen et al., 2011). The advent of the AQ uniquely provides a means for quantitatively assessing autistic traits in the typical population, and gaining insights into the individual differences that might predict perspective-taking abilities.

In the present work, we administered the $A Q$ to a sample of typical adults and assessed their VPT ability using a task that distinguishes between VPT-1 and VPT-2. If autistic traits in the typical population differentially predict VPT-1 versus VPT-2 ability, then individuals with higher AQ should show performance differences on trials targeting VPT-2, but not VPT-1. To converge with the extant literature, we also assessed whether gender would modulate VPT-1 and VPT-2 performance. We expected that AQ may prove a stronger predictor of male relative to female VPT-2 performance. Such results would contribute to knowledge regarding the cognitive mechanisms involved in perspective taking, and the individual differences that modulate the ability to temporarily adopt another's visual perspective. 


\section{Method}

\subsection{Participants}

One hundred and forty undergraduates ( 77 females, age $M=19.9, S D=1.8 ; 63$ males, age $M=19.8$, $S D=2.1$ ) who did not meet the diagnostic criteria for psychiatric disorders provided informed consent. The majority of participants self-identified as Caucasian $(n=121)$, six as African American, and nine as Asian (four opted to remain undisclosed).

\subsection{Materials}

We used the mini Structured Clinical Interview for DSM-IV to screen for Axis-I clinical diagnoses (mini-SCID), allowing for a reasonable assurance that our sample represented a non-clinical population.

We administered the self-report $A Q$ to measure individual differences in autistic traits (Baron-Cohen et al., 2001b). The AQ contains 50 questions assessing social skills (e.g., I enjoy social occasions), attention switching (e.g., I prefer to do things the same way over and over again), attention to detail (e.g., I often notice small sounds when others do not), communication (e.g., I enjoy social chit-chat), and imagination (e.g., I find making up stories easy). Participants respond using a four-point scale with anchors Definitely Agree, Slightly Agree, Slightly Disagree, and Definitely Disagree. Validation studies (e.g., Baron-Cohen et al., 2001b and Wakabayashi et al., 2006) demonstrate high test-retest reliability $(r=.70)$, and moderate to high internal consistency (Cronbach's alpha $\left.=.63^{-} .77\right)$ in both normal and autistic samples.

A VPT task (Kessler \& Thomson, 2010) used a series of images depicting an avatar seated at a circular table at each of six angle positions $\left(60^{\circ}, 110^{\circ}, 160^{\circ}, 200^{\circ}, 250^{\circ}, 300^{\circ}\right)$ arranged in a clockwise manner (Fig. 1). In the center of the table is a small barrier that is either perpendicular to (VPT-1) or parallel to (VPT-2) the facing direction of the avatar. There were four images for each of the six angle positions, two of which depicted a small red light either visible or hidden from the avatar (in front of or behind the barrier; VPT-1), and two depicted the light to the left or right of the barrier (VPT-2). The VPT-1 task involves simply following the avatar's line of sight to determine whether he can see an object or not; the VPT-2 task involves adopting the avatar's perspective in order to determine how the object looks from his unique perspective. The VPT-2 task becomes more difficult at higher angular deviation from the participant's orientation $\left(0^{\circ}\right)$, as demonstrated by increasing response times (Kessler \& Thomson, 2010). The VPT-1 task, in contrast, does not vary in response times as a function of angular deviation. 


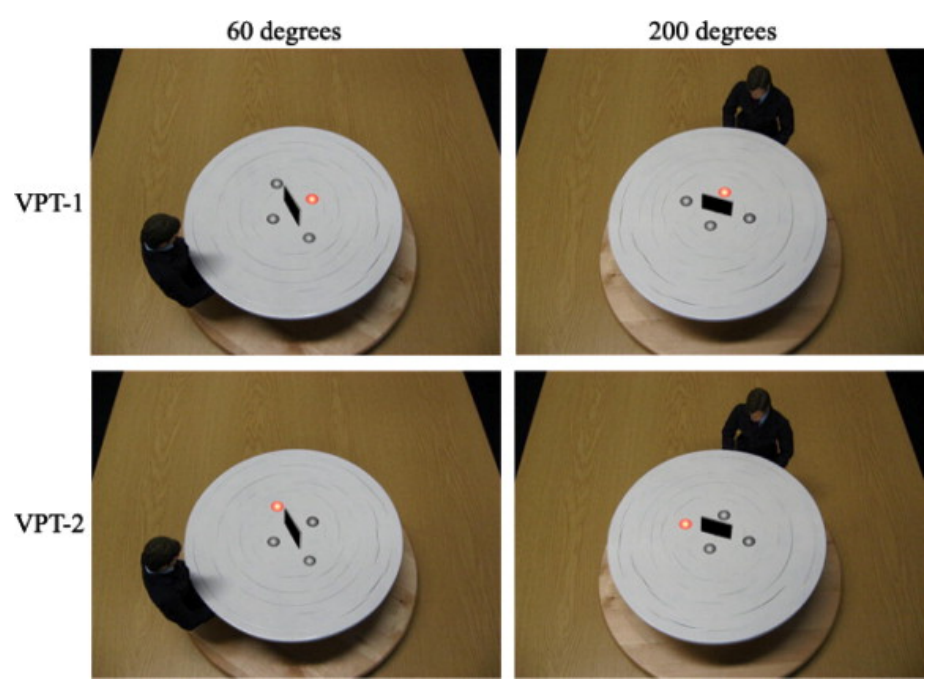

Fig. 1.

Sample VPT-1 (top row) and VPT-2 (bottom row) trials at $60^{\circ} \quad$ (left column) and $200^{\circ} \quad$ (right column). The upper left image depicts a hidden VPT-1 trial, upper right a visible VPT-1 trial, lower left depicts a left VPT-2 trial, and lower right a right VPT-2 trial.

\subsection{Procedure}

Participants first completed the computerized mini-SCID and AQ, and then began the VPT task. After a brief practice session, 14 blocks alternated between sets of VPT-1 (visible/hidden) and VPT-2 (left/right) tasks, with each block containing four sets of 12 trials (two trials per angular position), for a total of 672 trials. A 500 ms central fixation cross appeared between each successive trial.

Participants were instructed to press labeled keys to respond to either whether the light was visible or hidden from the avatar's view (VPT-1), or whether the light was to the avatar's left or right (VPT2), as quickly and accurately as possible. If a participant did not respond to a trial within $5 \mathrm{~s}$, the trial would terminate and move on. Whether the first block was VPT-1 or VPT-2 was balanced across participants, and trials were randomly presented within each set. No formal breaks were provided, though participants were given instructions prompts prior to each successive block, during which they typically paused for 5-10 s prior to continuing. Accuracy and response time data were automatically compiled by the SuperLab 4.0 (Cedrus Inc.) software. Overall, participants took approximately 10-15 min to complete the VPT task.

\section{Results}

\subsection{Scoring}

The mini-SCID resulted in the removal of 21 participants due to self-reported depression, mania, delusions, and/or hallucinations. Seven participants were removed due to near-chance accuracy on the VPT task (<65\%; range 52-63\%; $M=57.2 \%$ ), which in all cases seemed to involve difficulty distinguishing right from left across all VPT-2 angles.

The AQ was scored using the method described by Austin and colleagues (i.e., Austin, 2005 and Stewart and Austin, 2009), which uses raw Likert-based 1-4 responses (rather than 0-1 coding), and reverse-scoring as necessary (i.e., Baron-Cohen et al., 2001b). Scores were 
approximately normally distributed (Fisher, 1930; skewness $=-.06$ ), ranging from 123 to 167 ( $M=146.1, S D=10.3$, median = 146). Internal consistency was high, with an overall Cronbach's Alpha of .71. AQ scores were similar across the two genders: females showed a mean of 145.1 (range 120-167), and males a mean of 146.1 (range 117-185).

AQ scoring using the conventional 0-1 procedure confirmed that all participants were below the clinical (i.e., clinically relevant autism spectrum disorder) threshold $(<32)$ defined by Baron-Cohen et al. (2001b).

\subsection{Analyses}

Accuracy was high overall on both the VPT-1 $(\mathrm{M}=.998, \mathrm{SD}=.006)$ and VPT-2 $(\mathrm{M}=.999, \mathrm{SD}=.005)$ tasks. Response times exceeding an individual's mean $\pm 2.5 \mathrm{SD}$ were removed before analysis $(2.1 \%$ of all data). Overall response time data as a function of VPT Task and Angle are detailed in Table 1. To assess gender and AQ effects on VPT, analyses proceeded in two steps. First, we conducted an omnibus analysis of covariance (ANCOVA) with AQ scores as a covariate in a 2 (Task: VPT-1, VPT2) $\times 6$ (Angle: $\left.60^{\circ}, 110^{\circ}, 160^{\circ}, 200^{\circ}, 250^{\circ}, 300^{\circ}\right) \times 2$ (Gender: Male, Female) mixed design. We then conducted a series of linear regressions using $A Q$ scores as predictors, with response times on VPT-1 and VPT-2 tasks as dependent measures, separately for males and females.

Table 1.

Mean and standard deviation response times during the VPT-1 and VPT-2 tasks, at each of the six angles.

Angle

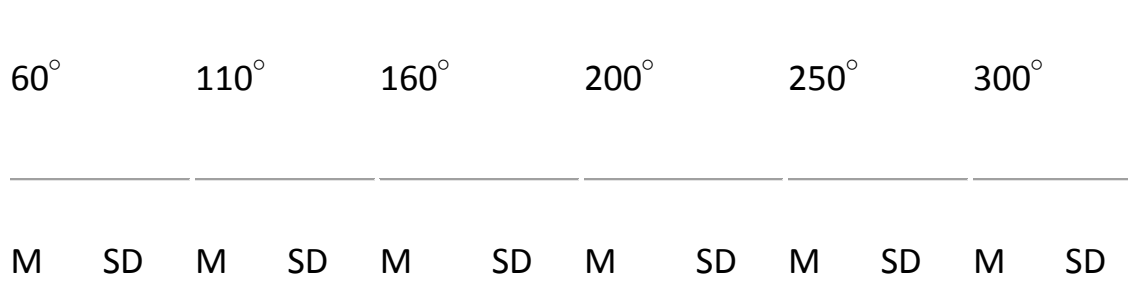

VPT-1

Males $\quad 735.982 .3 \quad 708.880 .5 \quad 713.4 \quad 81.6 \quad 728.6 \quad 85.9 \quad 741.180 .2 \quad 737.689 .7$

Females $722.871 .4 \quad 705.575 .3 \quad 702.0 \quad 79.2 \quad 720.1 \quad 77.1 \quad 728.274 .9 \quad 727.973 .8$

VPT-2

Males $\quad 833.5215 .1877 .1202 .91034 .8216 .41004 .9237 .3888 .9224 .2810 .2185 .5$

Females $775.9200 .4829 .4223 .1966 .8 \quad 287.9904 .7 \quad 251.9826 .3245 .6766 .4186 .8$ 


\subsection{ANCOVA}

The omnibus ANCOVA revealed a main effect of Angle, $F(5,545)=4.49, p<.01, \eta 2<.01$, and two 2way interactions, one between Angle and $A Q, F(5,545)=8.87, p<.01, \eta 2=.01$, and another between Angle and Task, $F(5,545)=4.54, p<.01, \eta 2<.01$. These effects were qualified by a 3-way interaction between Angle, Task and $A Q, F(5,545)=8.08, p<.01, \eta 2=.01$, and a 4-way interaction between Angle, Task, $A Q$ and Gender, $F(10,545)=4.45, p<.01, \eta 2=.01$.

To follow-up on the 4-way interaction, we conducted two separate 6 (Angle) $\times 2$ (Gender) $\times A Q$ mixed ANCOVAs, one for each VPT Task. For VPT-1, there were no main or interactive effects (all $\left.p^{\prime} s>.05, F^{\prime} s>1.4\right)$. For VPT-2, there was a main effect of Angle, $F(5,540)=7.08, p<.01, \eta 2=.05$, and two 2-way interactions, one between Angle and $A Q, F(5,540)=11.19, p<.01, \eta 2=.09$, and one between Angle and Gender, $F(5,540)=2.5, p<.05, \eta 2=.02$. These effects were qualified by a 3-way interaction between Angle, $A Q$, and Gender, $F(5,540)=2.31, p<.05, \eta 2=.02$.

To follow-up on the 3-way interaction within VPT-2, we conducted two separate ANCOVAs with Angle and $A Q$ as factors, one within Males and one within Females. For Males, there was a main effect of Angle, $F(5,165)=6.03, p<.01, \eta 2=.13$, and an Angle by $A Q$ interaction, $F(5,165)=7.87$, $p<.01, \eta 2=.17$. Females also showed an Angle by $A Q$ interaction, $F(5,375)=2.95, p<.05, \eta$ $2=.04$. Note, however, that $A Q$ was a stronger modulator of the Angle effect for Males ( $\eta 2=.17$ ) than for Females ( $\eta 2=.04$ ).

\subsection{Regressions}

To test the linearity and directionality of AQ's effect on VPT-2 performance as a function of Gender, we conducted a series of simple linear regressions. In all regressions, we used $A Q$ scores to predict the magnitude of the Angle effect $\left[\left(\mathrm{RT} 160^{\circ}+\mathrm{RT} 200^{\circ}\right) / 2-\left(\mathrm{RT} 60^{\circ}+\mathrm{RT} 110^{\circ}+\mathrm{RT} 250^{\circ}+\mathrm{RT} 300\right.\right.$ )/4].

Confirming the ANCOVA results, the first regression considered all participants and revealed significant predictive value of the AQ for VPT-2 (see Fig. $2 \mathrm{~b}$ ), $\beta$ std $=.37, \mathrm{t}(111)=4.15, \mathrm{p}<.01$, $\mathrm{R} 2=.14$, but not VPT-1 scores, $\beta \mathrm{std}=.06, \mathrm{t}(111)=.62, \mathrm{p}>.05, \mathrm{R} 2<.01$ (see Fig. 2a). In general, those with higher AQ showed a more dramatic Angle effect in VPT-2 than those with lower AQ. 


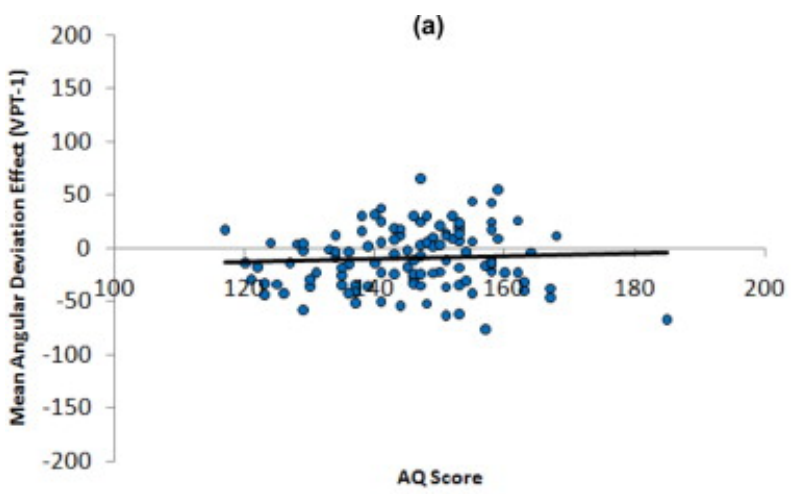

(b)

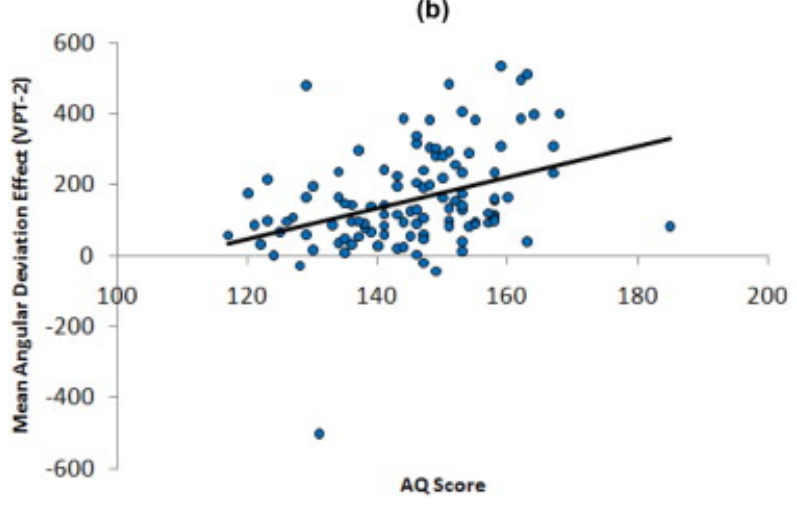

Fig. 2.

Regressions demonstrating the predictive value of the $A Q$ score in determining the mean angular deviation effect for VPT-1 (panel a) and VPT-2 (panel b).

The second and third regressions considered Males only, and then Females only. The Males showed significant predictive value of the AQ for VPT-2, $\beta$ std $=.52, \mathrm{t}(34)=3.51, \mathrm{p}<.01, \mathrm{R} 2=.27$, but not VPT-1 scores, $\beta$ std $=.22, \mathrm{t}(34)=1.32, \mathrm{p}>.05, \mathrm{R} 2=.05$. Female data patterned similarly, with significant predictive value of the $A Q$ for VPT-2, $\beta$ std $=.26, t(76)=2.36, p<.05, R 2=.07$, but not VPT-1 scores, $\beta$ std $=-.01, \mathrm{t}(76)=.08, \mathrm{p}>.05, \mathrm{R} 2<.01$. As expected, the predictive value of $A Q$ toward VPT-2 performance was nearly four times greater for Males $(R 2=.27)$ relative to Females $(\mathrm{R} 2=.07)$.

\section{Discussion}

The present study examined whether autistic personality traits in typical adults predict the ability to perform a visual perspective-taking task that differentially recruits VPT-1 and VPT-2 abilities, and whether these effects might be modulated by participant gender. Results suggest that sub-clinical autistic traits characterizing typical adults predict the ability to adopt another person's unique perspective. Specifically, though both low- and high-AQ individuals showed the typical pattern of increased response times as a function of angular deviation when performing the VPT-2 task, those with high AQ scores showed higher response times relative to those with low AQ scores. Interestingly, these results were most pronounced at high angular deviation, where the VPT-2 task is more likely to involve mentalizing and/or mental simulation of another's perspective. At the relatively low angular deviations the participant's self-referenced perspective is similar to the 
avatar's perspective, but higher angular deviations demand a decoupling of the two perspectives (Kessler, in press). In contrast, VPT-1 performance was not predicted by AQ scores, and did not vary as a function of angular deviation.

There was also evidence that the effects of AQ on VPT-2 performance are stronger for males than females. This result supports the notion that males may show lower fluidity in adopting another's visual perspective; this gender difference in visual perspective-taking may prove instrumental in the more general ability to understand what others see, think and feel (Baron-Cohen et al., 2001a, Baron-Cohen et al., 2001b, Eisenberg and Lennon, 1983 and Happe, 1995). Such a finding may reflect the lower frequency with which males versus females report adopting someone else's perspective during everyday situations (Davis, 1980 and Davis and Kraus, 1991). Note that, as detailed in the Method section, the present gender differences cannot be attributed to differences between males and females in terms of $A Q$ means or ranges.

These results contribute to the extant literature in three primary ways. First, we begin filling the knowledge gap concerning the individual differences predictive of the everyday cognitive feat of temporarily adopting another's perspective. Though perspective-taking has traditionally been viewed as requiring similar cognitive mechanisms as some basic visuospatial tasks such as mental rotation, more recent work has suggested that perspective-taking may be a relatively isolated cognitive process both behaviorally (Hegarty and Waller, 2004 and Kozhevnikov and Hegarty, 2001) and neurally (Ruby and Decety, 2001, Ruby and Decety, 2003 and Ruby and Decety, 2004). Given its ubiquity in daily life, there is a surprising paucity of empirical research aimed at isolating and characterizing the individual differences that might predict perspective-taking ability. We support the earlier survey-based (Davis, 1980) and experimental (Eisenberg \& Lennon, 1983) research suggesting that females show generally greater perspective-taking ability, and extend this work to show that females'versus males'ability to adopt perspectives is less vulnerable to individual differences in autistic personality traits.

Second, we validate the notion that autistic traits measured using the $A Q$ extend into the typical population and can be used to predict behavior on tasks characteristically impaired in individuals with ASDs (e.g., Baron-Cohen et al., 2001b). This recent approach to understanding clinical disorders by examining the predictive value of individual differences towards cognitive task performance and neural functioning in typical adults may prove valuable to the understanding of ASDs (e.g., von dem Hagen et al., 2011), and understanding the individual differences that predict task variance in typical adults. Finally, we contribute to theoretical dissociations between levels of visual perspective-taking by demonstrating differential predictive value of AQ in guiding VPT-1 versus VPT-2 abilities. Our results are congruent with work demonstrating separable cognitive and neural mechanisms responsible for the two VPT tasks, as supported by later evolutionary and developmental (e.g., Hamilton et al., 2009 and Tomasello et al., 2005) maturation of VPT-2 versus VPT-1 abilities.

Putting yourself in another's shoes in order to understand the world from their perspective is an exceedingly common task during social interactions; for instance, when describing where an object is relative to another (e.g., In front of you, to your left.) or giving another instructions on how to navigate (e.g., When you see the psychology building, turn left.) ( Brunyé et al., 2009 and Brunyé et al., 2008). Some work suggests that the ability to adopt another's visual perspective is an important precursor to successful empathizing, and also proves predictive of prosocial behavior such as 
helping, sharing and donating ( Underwood and Moore, 1982 and Yum and Schenck-Hamlin, 2005). Future work may attempt to disentangle the visual, cognitive and emotional components of empathizing that might be predicted by variation in autism spectrum traits. Overall, we demonstrate that gender, and autistic personality traits characterizing the typical population, may prove valuable in predicting a person's ability to perform an everyday visual perspective-taking task. These findings begin to specify the individual differences predictive of the ability for people to temporarily imagine themselves viewing the world from another person's perspective.

\section{References}

M. Aichhorn, J. Perner, M. Kronbichler, W. Staffen, G. Ladurner Do visual perspective tasks need theory of mind?

Neuroimage, 30 (3) (2006), pp. 1059-1068

E.J. Austin

Personality correlates of the broader autism phenotype as assessed by the Autism Spectrum Quotient (AQ)

Personality and Individual Differences, 38 (2005), pp. 451-460

S. Baron-Cohen

Perceptual role taking and protodeclarative pointing in autism

British Journal of Developmental Psychology, 7 (2) (1989), pp. 113-127

S. Baron-Cohen, S. Wheelwright, J. Hill, Y. Raste, I. Plumb

The 'Reading the Mind in the Eyes'Test-Revised version: A study with normal adults, and adults with Asperger syndrome or high-functioning autism

Journal of Child Psychology and Psychiatry, 42 (2001), pp. 241-251

S. Baron-Cohen, S. Wheelwright, R. Skinner, J. Martin, E. Clubley

The autism-spectrum quotient (AQ): Evidence from Asperger syndrome/highfunctioning autism, males and females, scientists and mathematicians

Journal of Autism and Developmental Disorders, 31 (2001), pp. 5-17

T.T. Brunyé, T. Ditman, C.R. Mahoney, J.S. Augustyn, H.A. Taylor

When you and I share perspectives: Pronouns modulate perspective-taking during narrative comprehension

Psychological Science, 20 (2009), pp. 27-32

T.T. Brunyé, D.N. Rapp, H.A. Taylor

Representational flexibility and specificity following spatial descriptions of real world environments

Cognition, 108 (2008), pp. 418-443

M.H. Davis

A multidimensional approach to individual differences in empathy

Catalog of Selected Documents in Psychology, 10 (1980), pp. 85-99

M.H. Davis

The effects of dispositional empathy on emotional reactions and helping: A multidimensional approach Journal of Personality, 51 (1983), pp. 167-184

M.H. Davis, L.A. Kraus 
Dispositional empathy and social relationships

W.H. Jones, D. PerIman (Eds.), Advances in personal relationships, Vol. 3JAI Press, Greenwich, CT (1991), pp. 75-115

N. Eisenberg, R. Lennon

Sex differences in empathy and related capacities

Psychological Bulletin, 94 (1983), pp. 100-131

\section{R.A. Fisher}

The moments of the distribution for normal samples of measures of departure from normality

Proceedings of the Royal Society, 130 (1930), pp. 16-28

J.H. Flavell, F.L. Green, E.R. Flavell

Development of knowledge about the appearance-reality distinction

Monographs of the Society for Research in Child Development, 51 (1986), pp. 1-87

U. Frith

Mind blindness and the brain in autism

Neuron, 32 (2001), pp. 969-979

A.F.D. Hamilton, R. Brindley, U. Frith

Visual perspective taking impairment in children with autistic spectrum disorder

Cognition, 113 (1) (2009), pp. 37-44

F. Happe

The role of age and verbal ability in the theory of mind task performance of subjects with autism

Child Development, 66 (1995), pp. 843-855

M. Hegarty, D. Waller

A dissociation between mental rotation and perspective-taking spatial abilities

Intelligence, 32 (2004), pp. 175-191

Kessler, K. (in press).

Spatial perspective taking is an embodied process, but not for everyone in the same way: differences for gender and autistic tendencies. Spatial Cognition and Computation.

K. Kessler, L.A. Thomson

The embodied nature of spatial perspective taking: embodied transformation versus sensorimotor interference

Cognition, 114 (1) (2010), pp. 72-88

M.D. Kogan, S.J. Blumberg, L.A. Schieve, C.A. Boyle, J.M. Perrin, R.M. Ghandour et al.

Prevalence of parent-reported diagnosis of autism spectrum disorder among children in the US, 2007

Pediatrics, 124 (2009), pp. 1-9

M. Kozhevnikov, M. Hegarty

A dissociation between object-manipulation and perspective-taking spatial abilities

Memory and Cognition, 29 (2001), pp. 745-756

K.P. Leith, R.F. Baumeister

Empathy, shame, guilt, and narratives of interpersonal conflicts: Guilt-prone people are better at perspective taking Journal of Personality, 66 (1998), pp. 1-37

T. Ohnishi, Y. Moriguchi, H. Matsuda, T. Mori, M. Hirakata, E. Imabayashi et al.

The neural network for the mirror system and mentalizing in normally developed children: An fMRI study

Neuroreport, 15 (9) (2004), pp. 1483-1487 
P. Ruby, J. Decety

Effect of subjective perspective taking during simulation of action: A PET investigation of agency

Nature Neuroscience, 4 (5) (2001), pp. 546-550

P. Ruby, J. Decety

What you believe versus what you think they believe? A neuroimaging study of conceptual perspective taking

European Journal of Neuroscience, 17 (2003), pp. 2475-2480

P. Ruby, J. Decety

How would you feel versus how do you think she would feel? A neuroimaging study of perspective taking with social emotions

Journal of Cognitive Neuroscience, 16 (2004), pp. 988-999

M.E. Stewart, E.J. Austin

The structure of the Autism-Spectrum Quotient (AQ): Evidence from a student sample in Scotland

Personality and Individual Differences, 47 (2009), pp. 224-228

J.P. Tangney

Shame and guilt in interpersonal relations

J. Tangney, K. Fischer (Eds.), The self-conscious emotions, Guilford Press, New York (1995), pp. 114-139

M. Tomasello, M. Carpenter, J. Call, T. Behne, H. Moll

Understanding and sharing intentions: The origins of cultural cognition

Behavioral and Brain Sciences, 28 (2005), pp. 675-691

B. Underwood, B. Moore

Perspective-taking and altruism

Psychological Bulletin, 91 (1) (1982), pp. 143-173

E.A.H. von dem Hagen, L. Nummenmaa, R. Yu, A.D. Engell, M.P. Ewbank, A.J. Calder

Autism spectrum traits in the typical population predict structure and function in the posterior superior temporal sulcus Cerebral Cortex, 21 (2011), pp. 493-500

A. Wakabayashi, S. Baron-Cohen, S. Wheelwright, Y. Tojo

The Autism-Spectrum Quotient (AQ) in Japan: A cross-cultural comparison

Journal of Autism and Developmental Disorders, 36 (2) (2006), pp. 263-270

Y.O. Yum, W. Schenck-Hamlin

Reactions to $9 / 11$ as a function of terror management and perspective taking

Journal of Social Psychology, 14 (3) (2005), pp. 265-286 